\title{
Research on social media information security questions in the big data era
}

\author{
Xiaoxing $\mathrm{Ma}^{1, \mathrm{a}}$ \\ ${ }^{1}$ Tianjin Hexi District Zhujiang Road 25\# TianjinUniversity of Finance \& Economics, \\ axxingl@163.com,
}

Keywords: Big Data, social media, risk management.

\begin{abstract}
Recent years social media swept the world, changed people's consumption behavior and lifestyle, and become a mainstream Internet applications. At the same time social media generate a lot of user data, let us be more realistic and comprehensive understanding of the structure, the personal information of the user than any previous era. However, risk management of information security on social media is a serious problem. Strengthening social media information risk management can establish and strengthen trust between users, between users and companies. The key of information risk management is the development and improvement of information risk and increase personal awareness of prevention and prevention methods, strengthen the construction of the Internet regulation laws, regulations and mechanisms.
\end{abstract}

\section{Introduction}

Although it is hard to believe, the first social media --Twitter appeared only less than a decade. Today, social media has become an important information platform for gathering, creating, sharing information. According to the report "China Social Media Impact Report" published by global survey group the Kantar Group: There is 36.4\% of the adult Internet users in China have concerned at least one social media platform. Social media have been as the main news and information supplement channel by more and more people. When the danger occurs, the user used to find information, broadcast propagation and contact person through the social network. News media even get image and video from Facebook, Tweeter, YouTube these social media as sightings resources and provide first-hand information, even do not after accurate verification. Undoubtedly, social media platform create a lot of possibilities for enterprises. But when users begin to focus on privacy management, the enterprise also brings a lot of challenges. Mobile social networking is not just wireless version of traditional desktop social networking, but more dynamic and interactive features, more content-rich, personalized social networking applications.

\section{Big data from social media}

Social media swept the world, at the same time generate a lot of user data, how to make effective use of these social data mining and become the most popular topic. Leverage social media data, can improve the ability to understand new phenomena, to achieve business intelligence, provide users with better services, and automatically discover innovative business opportunities. For example, social data mining to identify people with important influence in the Internet social environment; discover and classify different interest groups hidden in the social networking sites; identifying a particular user and according to user's subjective feelings at a time, develop active commodity purchase recommendation system and a friend recommended application; mastered the process of evolution and change for IP networks entity-relationship; protect social network users' privacy and security; establishing and strengthening trust between users and the user with the entity. Big data of social media also allows us more realistic and comprehensive understanding of the structure, interest, motivation and the consumption characteristics of users than any previous era, and on this basis can 
really make precision marketing even customized marketing possible. Meanwhile, the "relevance" logical thinking of big data in social network of keyword analysis, offers unlimited creative space to implanted marketing value chain extension. Data mining of social media is a vibrant interdisciplinary field, scholars of different disciplines for social media development has made tremendous contributions.

Our social media platform is rapidly expanding and upgrading, according to the social applications of mobile equipment installation league released on September 2014, social media can be approximately divided into six categories [Table 1]. Popularity of social media applications to produce a variety of social media data, such as text stream data from Weibo ,multimedia data from multimedia sharing sites, user interaction data from social networking site, location data from sign site, consumption data from online shopping sites. These big data of social media recorded from different angles of people's online life, and mapping the links between people in the real world.

Table 1 Social Media Category

\begin{tabular}{|l|l|}
\hline \multicolumn{1}{|c|}{ Category } & \multicolumn{1}{|c|}{ Platform } \\
\hline $\begin{array}{l}\text { Online social networking } \\
\text { site }\end{array}$ & Sina Weibo, Tencent Weibo, QQ space \\
\hline Social news & Phoenix news \\
\hline Blog & Sina blog, Sohu blog \\
\hline Dating & Momo \\
\hline Ranking Reviews & Dianping, Mtimes \\
\hline Media Sharing & Tudou, Douban \\
\hline Instant messaging & Wechat, QQ \\
\hline
\end{tabular}

Research on multi-source phenomenon of social media, understanding multi-source data of social media, data mining of social media is an important step of big data. It's important for the discovery of new discipline, more comprehensive use of social media data, analysis and design of complex social media applications. For example, the information flow micro blogging site, the social networking site reviews, media sharing sites, pictures and video analysis, you can track the progress and evolution of an event from different angles; associated the video viewing data of people in the video sharing site and consumption data from online shopping sites, helps to understand people's interest and consumption model, and applied to targeting marketing activities.

Different social media concern in different areas of services, in order to enjoy a variety of services, people will also participate in the different sites. For example, people to keep in touch with friends in Renren website, concern and tracking hot events in Weibo, share your favorite music, movies, books in Douban, share reviews about restaurants and food images in Dianping ranking. We call the same person shared one user account for different the social media networks across-the- network user. The behavioral of same person at different information network provides the possibility to analyze and use social media data from multiple sources. On the one hand, the user is the main target of social media data analysis, applicant integration on user behavior information scattered in different social media networks is important for in-depth understanding of user; we dig multiple sources of data associated with social media knowledge for collaborative analysis and application to provide a solution based on a common user across the network, we explicate both in across-the- network user modeling and associated user data mining from multiple sources of knowledge.

\section{Types and causes of information risk lead by social media}

\section{Location information in the social media}

Social media is not just the mobile version of traditional social networks, need for more in-depth and accurate classification and research base on its characteristics. Location-based applications are the typical social media, and thus a threat to the privacy of the very large. Through social media application, users can not only know their own position, but also through the push function to find friends from nearby location. Network share location information makes social media applications wider than traditional web platform, installed in the smart device version of social media applications 
allow users to use mobile devices at any time to locate and update your location information. After the user finished the text with photos or video sharing, published content can be select to add the precise location information. Location information is an important privacy. First, the location information is disclosed to increase the threat to the user is tracked. Secondly, according to the analysis of continuous and recurring location information can judge privacy such as work location, home address and health status. In addition, free will of user to hide the true location therefore subject to restrictions.

Network share location information makes social media applications more frequently than traditional web platform applications, smart devices installed in the version of social media applications allow users to use mobile devices at any time to locate and update your location information. You can search for people in the vicinity of the location information through the use of Weibo, Wechat and other applications to add new friends, to provide people with the opportunity to be close to strangers. These applications facilitate better communication between people in the same area, but the information resulting from the terrible risk.

\section{Information risk triggered by social media platform operators}

Social media has become an inseparable part of everyday life for users. The reason why users ignore privacy settings, the first is the lack of awareness of privacy protection; secondly, irrational privacy settings function of social media application will become a major motivation of privacy leakage. From the aspect of the settings about association user accounts of social media, many privacy function setting of social media are not reasonable, most of the social media platform is target to the user's convenience, allows user register with associate user account from different platforms is an important aspect of information risks. For example, users can use their own Weibo accounts, QQ accounts, to login other website or social application, after account binding, the content published in the other website will automatically sync to user's Weibo and QQ. This is because the carriers use associated account, although it can omit user registration operation, but when the user account information of one platform being stolen, associated account of other social media were all faced with the risk of information leakage. And because the associated account mechanisms, their friends information on different social media platforms will also be collected and used by other social media companies. Even if the user selects the most advanced the settings in privacy protection, companies can also easily infer the user's personal information based on the information of users and friends.

Some social media companies collect user personal information too much, and most social media companies using a privacy protocol to disclaimer for collect and share information on user behavior information. For privacy protocol, users tend to click on the "Read" or "agree" button, but when once user information is illegal to provide to other organizations, it is possible caused great harm for the user's personal privacy, and the user will not safeguard their rights as they has been signed these privacy agreements.

While many users have realized the problems caused by privacy leak, and companies ran social media provides users with privacy settings, but studies show that a lot of users are not using these privacy setting options, it defaults setting to open to all, and about 1300 million users said they never set, or simply do not know privacy settings. $28 \%$ of users share most or all of their content. Users fill in the information and update status on social media it is mainly based on their social motives. Users want to keep in touch with friends and family through social media, but also hope to find friend for many years without contact through automated recommendation function of social media, or find a new friend of common social circle or hobbies, to expand their social circle. The information more complete and it is likely to get a more accurate recommendation.

In addition, some network service providers have not fulfilled responsibility for information security about store user information. June 2013 China Software Testing Center issued a "Site safety evaluation report" shows: In 100 sites, only eight companies to take higher level security measures; nearly $60 \%$ site companies do not take safety measures to protect the user information, and even user passwords are stored in plain text, so that the user's password is directly exposed to the network, it is 
easy to steal personal information from hackers. Exposed awareness that many social media platforms companies for user privacy information protection is not strong.

\section{Social media violations of privacy caused}

Social issues, hot issues arouse public attention; many users will even vent their emotions through social media. Every hot topic will cause large-scale search and screen of user information. In big data environment, data mining data becomes very easy for the mass storage of personal information and behaviors, over a night, all living information of who is the target will be made open to public. This is a violation of personal privacy, and big data of social media is the driving force behind for such violations.

"Tweet a Tweet", "Push Facebook" has become necessary daily activities for mobile Internet users. The characteristic that immediate and mobility of mobile social media, will emerge the user's private information may every minute to others. According to estimates of the National Research Center of Consumer Reports, there are 480 million people use Facebook to tell people where to go someday, which is kind of useful clues for thief; there are 470 million "Like" about the health condition or treatment on Facebook, this information may be used by insurance salesmen.

\section{Information risk management program of social media}

According to China Internet Network Information Center, the "2014 Chinese social networking application user behavior research report" shows that instant messaging applications coverage reached $89.3 \%$ in overall Internet users, social networking site's coverage $61.7 \%$, Weibo's coverage is $43.6 \%$, social networking has been the third largest information dissemination channels. According to the 2014 annual China Internet security report shows that in 2014, the proportion of fishing attacks spread by social media reached $20.4 \%$, social media has become the next target group which hacker attacks after the online game, the online shopping website. In big data environment, government should promulgate appropriate policies and regulations to assist those organizations using data generated social media, to assume responsibility for information risk prevention, in order to circumvent the risk brought by big data of media information technology.

\section{To strengthen legislation and industry regulations}

At present, government introduced the personal information about social media safety laws and regulations in the "Criminal Law Amendment (7)", but only a single article provisions, also need assistance to introduce the conviction and sentencing of judicial interpretation, cases also shows that operability is not strong in practice, can not be unified for execution of similar cases, the situation are incompatible which is the rapid development of social networking applications. The introduction of laws and regulations to protect the user information social media platforms, China needs to learn from the experience of other developed world, legislation to establish a scientific and detailed rules for the protection of personal information security as soon as possible, clearly defined the body and the boundary about the rights and obligations on social media

From the industry level, the supervision of trade associations is a way to solve the policy is lagging behind, industry regulation mechanism is an indispensable part of social media user's information risk prevention system, social media companies should accept the supervision of trade associations, take protection for user privacy responsibilities actively. With the popularity of social media, the user information risk management will become more valuable. Social media companies should increase its investment in the user information storage, privacy settings, user information and other aspects of security warning.

\section{To improve information security management of social media operators}

Social media companies are first responsible person of the security of user information, in the case against leakage about the user information, the companies in the improper proportion is increasing. Social media companies usually tend to listed disclaimer in the "Terms of Use", "Conditions of Service" or "Terms and Conditions" agreement, but in fact such clauses is not able fully indemnified, this approach violates the choose right for the user's to. Most social media companies, on the one hand they use reward points, priority to use of the new features and other ways to attract users to fill in 
user information, such as name, home address, e-mail address, phone number and QQ number. On the other hand did not pay attention to security risk management of user information, do not invest enough funds to build a professional information security management team, ignoring the user's information security risks. Social media companies should therefore be aware the importance of risk management of user information, fully implement their duties, in order to prevent information security risks, take the risk management of user information as an important part of enterprise information security management, take efforts to improve management.

\section{Enhance risk awareness information of social media users}

Risk awareness means that under big data, an information security knowledge, ethics and information security capabilities, such as integrated together, information security risks identified and the user has the ability to guard their user information on social media applications, It should be noted that most social media applications require users to real-name registration, so authenticity of the user information is high, the user's friends are basically familiar, so will reduce the users awareness of risk on the platform, users often blindly follow the news and information friends share through social media without judgment.

Users who often release a lot of photos and videos of everyday life would be more high risk to information leaks. Using social media search function can easily get some personal information, if combination of big data search engine, then it is very easy leaked to sensitive personal information. The user can not assume social media platform provides privacy protection clause, it will provide good protection for the privacy of users of information security, users should thoughtful when publish and share information, sensitive information should not be published on the social media platform, to prevent information will be collected by malicious people for fraud or commercial promotion.

\section{Conclusion}

Social media has become an important tool for people to communicate. In big data environments, people use the social media to create value but also constantly to exchange personal information, information risk management to protect user information security is facing a severe test and challenge, security risk management about personal information has become an important issue relating to the vital interests of each network user, the information risk on social media and other Internet application is a serious major problem that must be faced in contemporary Chinese.

The rise of social media breaks the boundaries between the virtual reality and the Internet, making opportunity for cybercrime invasion for personal privacy. Users use social media to generate massive big data, big data era coming, and if the information risk governance not good, will severe blow to user confidence about the social media, and ultimately affect the healthy development of social media and the Internet fundamentally, subsequently affect healthy development of the Internet industry.

The key of information risk management is the development and improvements, and increase personal awareness of prevention and prevention methods, strengthen the construction of the Internet regulation laws, mechanisms, efforts from the technical and management aspects, governance information risk better, make protection measures are in place to ensure the security of personal information the user can be protected.

\section{References}

[1] Xulin Jia, The dissemination of research content and methods of influence. - Reflection on social media and mobile Internet for big data, (2003).

[2] Jian Meng, Liu Yichuan, Big Data era: on change of media production - Based on the Perspective of Media Economics, [J].Journalism and Communication, (2003).

[3] Dan Webber, From a push-off to the National Social media crisis _ Risk Management [J], Food Safety Guide, 2014. 
[4] Xiaofeng Meng, Zhangxiao Zhong, Big Data privacy management [J],Computer Research and Development, 2014 (10).

[5] Zhang Meng, Li Yang, Based on PCA and data model of the gravitational field of social media information Confidence Evaluation Method [J], Microcomputer, 2014 (30-9).

[6] Information on http://h.cnnicresearch.cn/download/report/rid/144

[7] Information on http://www.isc.org.cn/wzgg/listinfo-29087.html

[8] Wen-ying Chang, Weibo Liu, Users divulge personal information based on the reliability analysis of empirical research - Sina Weibo[J], Intelligence magazine, 2015 (34-8).

[9] Qiang She, Using social networks and avoid risks it brings [J]. Information Security and Communications Privacy, 2011 (10). 